\title{
Correction
}

\section{Correction to: On Semi-finite Hexagons of Order $(2, t)$ Containing a Subhexagon}

Anurag Bishnoi and Bart De Bruyn

\section{Correction to: Ann. Comb. 20 (2016) 433-452 https://doi.org/10.1007/s00026-016-0315-z}

In Section 4.2 of [1], we showed that there does not exist any infinite near hexagon $\mathcal{N}$ of order $(2, t)$ that contains an isometrically embedded subgeometry $\mathcal{H}$ isomorphic to $H(2)$. The proofs of Lemmas 4.6 and 4.7 in [1] have been spoiled by the same error: points of $\mathcal{N}$ at distance 1 from $\mathcal{H}$ are not necessarily collinear with a unique point of $\mathcal{H}$ (see Page 446, Line -8 and Page 447, Line 2). This is true in case $\mathcal{N}$ is a generalised hexagon, but not if $\mathcal{N}$ is a general near hexagon. Luckily, these errors can be corrected.

The following proof should replace the proof of Lemma 4.6 in [1].

Lemma 1. There are only finitely many points of type $B_{1}$ in $\mathcal{N}$.

Proof. Let $\mathcal{B}$ denote the set of those points of $\mathcal{N}$ that have type $B_{i}$ for some $i \in\{2,3,4,5\}$. Then $\mathcal{B}$ is finite by [1, Lemma 4.5]. Let $\mathcal{A}$ denote the set of those points of $\mathcal{N}$ that have type $A$, i.e., the points of $\mathcal{H}$. Then the set $\mathcal{A} \cup \mathcal{B}$ is also finite. Let $x$ be a point of type $B_{1}$ in $\mathcal{N}$. Then by [1, Lemma 4.2], $x$ is at distance 1 from $\mathcal{H}$, and since $\mathcal{O}_{f_{x}}$ is a singleton, there exists a unique point $\pi(x)$ in $\mathcal{H}$ collinear with $x$. If $x$ is only collinear with points of type $A, B_{1}$ or $C$, then by the same reasoning as in the proof of [1, Theorem 4.4], we get a contradiction. So, $x$ is collinear with at least one point of $\mathcal{B}$, and we have already seen that it is collinear with at least one point of $\mathcal{A}$. Thus $x$ is the common neighbour of two points at distance 2 in the finite set $\mathcal{A} \cup \mathcal{B}$. Since each such pair of points at distance 2 in the near polygon $\mathcal{N}$ has finitely many

The original article can be found online at https://doi.org/10.1007/s00026-016-0315-z. 
(at most five) common neighbours, we see that the set of points of type $B_{1}$ must be finite; in fact, the cardinality of this set is bounded by five times the number of unordered pairs of points at distance 2 in $\mathcal{A} \cup \mathcal{B}$.

The following proof should replace the proof of Lemma 4.7 in [1].

Lemma 2. There are only finitely many points of type $C$ in $\mathcal{N}$.

Proof. Let $x$ be a point of type $C$ in $\mathcal{N}$. Then the set of points of $\mathcal{H}$ at distance 2 from $x$ is a 1-ovoid of $\mathcal{H}$ and hence it has cardinality 21 . Let $S_{x}$ be the set of common neighbours between $x$ and the elements of $\mathcal{O}_{f_{x}}$ (the 1-ovoid of $\mathcal{H}$ induced by $x$ ). By [1, Lemma 4.2], each element $y$ of $S_{x}$ has type $B_{i}$ for some $i \in\{1,2, \ldots, 5\}$ and hence by [1, Table 3] $y$ is collinear with at most nine points of $\mathcal{H}$. Therefore, $\left|S_{x}\right| \geq \frac{21}{9}$, and we get two points of the set $\Gamma_{1}(\mathcal{H})$ at distance 2 from each other having $x$ as a common neighbour. By [1, Lemma 4.5] and Lemma 1 , the set $\Gamma_{1}(\mathcal{H})$ is finite. A similar reasoning as in the proof of Lemma 1 then shows that there are only finitely many points of type $C$ in $\mathcal{N}$.

The rest of the discussion in Section 4.2 of [1] can remain as it is. In the proof of Lemma 4.3 , there is however a typo. The condition $\mathrm{d}\left(x, y_{1}\right)=$ $\mathrm{d}\left(x, y_{2}\right)=\mathrm{d}\left(x, y_{3}\right)$ should be replaced with $\mathrm{d}\left(y, x_{1}\right)=\mathrm{d}\left(y, x_{2}\right)=\mathrm{d}\left(y, x_{3}\right)$.

Publisher's Note Springer Nature remains neutral with regard to jurisdictional claims in published maps and institutional affiliations.

\section{Reference}

[1] Bishnoi, A., De Bruyn, B.: On semi-finite hexagons of order $(2, t)$ containing a subhexagon. Ann. Comb. 20(3), 433-452 (2016)

Anurag Bishnoi and Bart De Bruyn

Department of Mathematics

Ghent University

Krijgslaan 281

9000 Gent

Belgium

e-mail: bart.debruyn@ugent.be

Anurag Bishnoi

e-mail: anurag.2357@gmail.com 